International Mathematical Forum, Vol. 9, 2014, no. 16, 763 - 766

HIKARI Ltd, www.m-hikari.com

http://dx.doi.org/10.12988/imf.2014.4346

\title{
For Each Mathematical Statement, Only Finitely Many of its Generalizations are Useful: A Formal Proof of E. Bishop's Idea
}

\author{
Olga Kosheleva and Vladik Kreinovich \\ University of Texas at El Paso \\ $500 \mathrm{~W}$. University \\ El Paso, TX 79968, USA
}

Copyright (c) 2014 Olga Kosheleva and Vladik Kreinovich. This is an open access article distributed under the Creative Commons Attribution License, which permits unrestricted use, distribution, and reproduction in any medium, provided the original work is properly cited.

\begin{abstract}
Generalization is one of the main mathematical activities. Some generalizations turn out to be useful for working mathematics, while many other generalizations have so far been not very useful. E. Bishop believed that most fruitless-so-far generalizations are hopeless, that every mathematical statement has only a few useful generalizations. In this paper, we show that, under a natural definition of the notion of useful generalization, Bishop's belief can be proven - moreover, it turns out that for each mathematical statement, only finitely many of its generalizations are useful.
\end{abstract}

Mathematics Subject Classification: 03A05

Keywords: useful generalizations, mathematics, proofs

\section{Formulation of the Problem}

Generalizations or quasi-generalizations? Generalization is one of the main mathematical activities, this is how many mathematical theories 
emerged: by realizing that, e.g., many results about real numbers hold for general fields or even for general rings. Many such generalizations have indeed turned out to be very useful.

On the other hand, many generalizations have not been very fruitful for working mathematics. About such generalizations, there are two opinions:

- Some mathematicians optimistically believe that many of these not-yetfruitful generalizations - and many yet-to-be-discovered future generalizations - will become fruitful some day. In the opinion of some of these mathematicians, there is no limit on how many times each mathematical result can be fruitfully generalized.

- On the other hand, other mathematicians strongly believe that very few generalizations are (or will be) fruitful. Other generalizations are (and will be) quasi-generalizations, i.e., generalizations which are of no use to working mathematics.

This second view was strongly supported by E. Bishop [1]; see also [2, 3]. Bishop's opinion became widely known since it was one of the main motivations why, after becoming a renowned prize-winning mathematician, he decided to concentrate on constructive mathematics, i.e., on the analysis of which mathematical objects are computable - and which existence results can lead to computability of the corresponding objects [1].

At first glance, this question seems to be un-answerable. At first glance, the above question may sound like one of the questions for which we cannot provide the answer now, like will the 22 century be the century of further progress or the 20-century-type era of mass human suffering: the only way to answer this question is to wait and see what will actually happen.

We will nevertheless provide an answer. In this paper, we show that, in contrast to a question about the suffering in the 22 century, we can answer the question about generalizations and quasi-generalizations.

Our answer supports Bishop's pessimistic opinion. Specifically, we will show that, within a reasonable formalization of the above questions, it can be proven that each mathematical statement has are only finitely many useful generalizations. This means that the vast majority (to be more precise, all but finitely many) of possible generalizations (current and future) are useless (i.e., are, in Bishop's terms, quasi-generalizations).

\section{Definitions and the Main Result}

How to define what is a useful generalization? Main idea. To formulate the problem in precise terms, we need to formally define what is a useful generalization. 
Intuitively, a statement $B$ is a useful generalization of a statement $A$ if it is easier to derive $A$ from $B$ than to derive $A$ "from scratch" (i.e., from the axioms).

Discussion. Of course, this is only one of the properties which are intuitively associated with useful generalizations. Probably, not every pair of statements satisfying the above property would be naturally called a useful generalization - but our claim is that every pair which is naturally called a generalization satisfies the above property.

Thus, if we prove that for every statement $A$, there are only finitely many "useful generalizations" in the sense of the above definition - this would imply that there are only finitely many statements $B$ which can be naturally called useful generalizations of $A$.

Definitions and the main result. Now, we are ready to formulate our main result.

Let us fix a formal theory $T$, with its alphabet, syntax, notion of a statement, axioms, and notion of a proof. In the following text, by statements and proofs, we mean statements from this theory and proofs in this theory.

Let us also fix a way to represent symbols from this language (and proofs from this language) in a computer. As a result, we will be able to represent each statement and each proof in a computer, i.e., we will be able to represent each statement and each proof as a sequence of bits (0s and 1s). By the length of a statement or a proof, we mean the total number of bits in (the computer representation of) this statement or proof.

Definition. We say that a provable statement $B$ is a useful generalization of a provable statement $A$ if $\operatorname{pr}(A \mid B)<\operatorname{pr}(A)$, where:

- $\operatorname{pr}(A)$ is the shortest possible length of a proof of $A$, and

- $\operatorname{pr}(A \mid B)$ is the shortest possible length of a proof of $A$ from $B$ (i.e., a proof of $A$ which, in addition to the original axioms, can also use the statement $B$ as a new axiom).

Proposition. For every provable statement A, there are only finitely many useful generalizations of this statement.

Proof. By definition, to each useful generalization, there corresponds a proof of $A$ from $B$ which is shorter than $\operatorname{pr}(A)$. The statement $B$ is a part of the formal proof which uses this statement $B$. Thus, the length of the statement $B$ itself - i.e., the number of bits in a computer representation of the statement $B$ - is also smaller than $\operatorname{pr}(A)$.

So, the number of useful generalizations $B$ cannot exceed the total number of binary sequences of length $<\operatorname{pr}(A)$. There are totally $2^{1}$ binary sequences 
of bit length $1,2^{2}$ binary sequences of binary length $2, \ldots$, and $2^{\operatorname{pr}(A)-1}$ binary sequences of length $\operatorname{pr}(A)-1$. Thus, the total number of such sequences is equal to $2^{1}+\ldots+2^{\operatorname{pr}(A)-1}=2^{\operatorname{pr}(A)}-2$. Therefore, there are finitely many such sequences and hence, finitely many useful generalizations of a statement $A$.

The proposition is proven.

Acknowledgments. This work was supported in part by the National Science Foundation grants HRD-0734825 and HRD-1242122 (Cyber-ShARE Center of Excellence) and DUE-0926721.

\section{References}

[1] E. Bishop, Foundations of Constructive Analysis, McGraw-Hill, New York, 1967.

[2] J. Kennedy and R. Kossack, Set Theory, Arithmetic, and Foundations of Mathematics: Theorems, Philosophies, Cambridge University Press, New York, 2011.

[3] A. S. Troelstra, "Proof theory and constructive mathematics", In: Mathematics: Concepts and Foundations, Vol. II, UNESCO and Encyclopedia of Life Support Systems EOLSS, Paris, France, 2001.

Received: March 12, 2014 